We describe the construction of a collection of quadrature formulae suitable for the efficient discretization of certain boundary integral equations on a very general class of two-dimensional domains with corner points. The resulting quadrature rules allow for the rapid high accuracy solution of Laplace's equation and the Helmholtz equation on such domains. Our approach can be adapted to many other boundary value problems as well as to the case of surfaces with singularities in three dimensions. The performance of the quadrature rules is illustrated with several numerical examples.

\title{
Universal quadratures for boundary integral equations on two-dimensional domains with corners
}

\author{
J. Bremer ${ }^{\dagger}$, V. Rokhlin ${ }^{\ddagger}$, and I. Sammis ${ }^{\dagger}$ \\ Technical Report YALEU/DCS/TR-1420
}

November 24, 2009

The first and third authors were supported by the Office of Naval Research under contract N0001409-1-0318. The second author was supported in part by the ONR under contract N0014-07-1-0711, in part by the AFOSR under contract FA9550-09 -1-0241, and in part by DARPA-AFOSR contract FA9550-07-1-0541.

$\dagger$ Dept. of Mathematics, Univ. of California, Davis, Davis CA 95616

$\ddagger$ Dept. of Mathematics, Yale University, New Haven CT 06511

Approved for public release: distribution is unlimited.

Keywords: Boundary integral equations, fast solvers, domains with corners, corner singularities. 


\section{INTRODUCTION}

In [4], a procedure for the construction of an orthonormal basis spanning the space of restrictions of functions satisfying a Laplace boundary integral equation over a contour $\Gamma$ to a small curve segment $\Gamma_{0} \subset \Gamma$ is introduced. Using the machinery of [3], such a basis can be exploited to form a "purposemade" quadrature formulae for the efficient discretization of the boundary integral equation over the curve segment $\Gamma_{0}$. As the size of the resulting quadrature rules depends only weakly on the geometry of the curve segment $\Gamma_{0}$, boundary integral equations over curves with complicated geometry (e.g., corners) can be discretized quite efficiently. Moreover, by repeating the basis construction procedure for multiple curve segments, a collection of quadratures suitable for the efficient discretization of a particular boundary integral equation over an entire class of contours with complicated geometry can be precomputed. We refer to such a collection as a set of "universal quadratures." The construction of universal quadratures for boundary integral equations arising from Neumann and Dirichlet Laplace boundary value problems on polygonal domains is described in [4]. The resulting quadrature rules achieve double precision accuracy with approximately 30 discretization nodes required per corner.

In the present paper, we introduce a modified version of the procedure of [4] applicable to boundary value problems for both Laplace's equation and the Helmholtz equation with small wavenumbers. We also describe the construction of collections of universal quadratures for the corresponding boundary integral equations on a very general class of domains with corners. The resulting quadratures allow for the rapid high accuracy solution of Laplace and Helmholtz boundary value problems on such domains.

A large number of schemes for the solution of boundary integral equations on domains with corners have been proposed, but unlike the work presented here, these techniques all suffer from one or more of the following serious disadvantages:

1. They require dense meshes of discretization nodes near corner points.

It is possible to discretize boundary integral equations near a singular point of the boundary by simply using a very dense quadrature. This practice, however, results in very large systems of linear equations in the case of domains with many corner points.

2. They involve classical quadrature techniques like subtraction of singularities, factorization, or substitution.

These techniques are simply too cumbersome to apply to large-scale domains. The behavior of a solution to a Helmholtz boundary integral equation with wavenumber $k$ near a corner point, for instance, can be described as

$$
\sigma(x)=\left\{\begin{array}{ll}
\eta(x)+\alpha(x, k) & \text { for } x<0 \\
\eta(x)+\beta(x, k) & \text { for } x>0
\end{array},\right.
$$

where $\eta$ is a singular function determined by the angle of the corner and $\alpha$ and $\beta$ are oscillatory functions whose behavior is dictated not only by the angle of the corner point but also the geometry of the boundary curve on either side of the corner point and the wavenumber of the Helmholtz equation. It might be that any particular case can be handled using classical quadrature techniques, but the number of modifications required in order to apply these techniques to the domain shown in Figure $5 c$ of Section 7, for instance, is already daunting and it is by no means a truly "large-scale" example.

Perhaps even more problematic is the lack of straightforward generalizations of these approaches to surfaces with singularities in $\mathbb{R}^{3}$.

3. They require the modification of the boundary integral equation.

For instance, many approaches for the solution of Laplace's equation on domains with corners take advantage of the fact that the second kind boundary integral equation over the contour $\Gamma$

$$
\pm \frac{1}{2} \sigma(x)+\int_{\Gamma} \frac{\partial}{\partial \nu_{y}} \log |x-y| \sigma(x) d S(y)=u(x)
$$


where $\partial / \partial \nu_{y}$ denotes differentiation in the variable $y$ with respect to the outward normal vector of $\Gamma$, is related to the Cauchy integral

$$
\int_{\Gamma} \frac{\sigma(z)}{z-w} d z .
$$

Operations involving the equation (1.1) can often be reduced to operations on the more tractable integral

$$
\int_{\Gamma} \frac{\sigma(z)-\sigma(w)}{z-w} d z,
$$

which is nonsingular when $\sigma$ is sufficiently smooth. Unfortunately, approaches of this type do not apply to many boundary integral equations of interest, and even when they do, they require adaptation to each new case. Moreover, this class of techinques does not readily generalize to three dimensions.

4. They require a priori analytical estimates of the singularities of solutions of the integral equation.

It is possible to exploit known analytical estimates characterizing solutions of boundary integral equations on singular domains (like those for domains with corners in [7]) to rapidly invert those equations. However, obtaining even those results known today has been a laborious process requiring great effort, and the current state of affairs is far from satisfactory. Estimates for planar domains are not yet comprehensive and results for surfaces are almost entirely lacking. Moreover, this approach suffers from the same fundamental problem as classical quadrature techniques; namely, it requires adaptation to individual cases which makes it cumbersome to use in practice.

Unlike previous approaches, our method does not rely on classical quadrature techniques and does not require dense meshes of discretization nodes near corner points, the modification of the underlying boundary integral formulation, or a priori analytical estimates of corner singularities. Rather, corner singularities are characterized numerically (via orthonormal bases) and efficient quadrature rules which obviate the need for dense meshes of discretization nodes are precomputed using those characterizations. The scheme generalizes easily to a wide variety of boundary value problems as well as to to the case of two-dimensional surfaces with singularities.

Our approach is most similar to the scheme proposed in [9], which appears to be the first paper describing the solution of large-scale problems on domains with corners, in which dense meshes of discretization nodes are coupled with a compression scheme for the resulting linear systems. The principal differences between the Helsing-Ojala algorithm and our scheme are: (1) our formalism (quadrature rules) allows for "compression" to be performed a priori at the time the quadrature rules are constructed rather than on-the-fly for each specific problem as in [9], and (2) the Helsing-Ojala scheme involves constructs which do not appear to readily generalize to the case of surfaces with singularities.

This paper is divided into seven sections. After dispensing with preliminary results pertaining to quadrature and interpolation in Section 2, we describe a very general Nyström framework for the discretization of integral equations in Section 3. In Section 4, we review the principal tool of [4], a procedure for the construction of a basis spanning the restriction of solutions of a particular boundary integral equation to a curve segment. The construction of quadratures for the efficient discretization of a single given curve segment is described in Section 5, while Section 6 describes a procedure for the construction of a collection of universal quadratures for a very general class of planar domains with corner points. Finally, numerical results are reported in Section 7.

\section{Generalized quadrature and interpolation}

In this section, when $X$ is an $n \times m$ matrix, we will denote by $\sigma_{j}(X)$ its jth largest singular value. Moreover, for $j>\min (n, m)$ we define $\sigma_{j}(X)=0$.

2.1. Discretization of square integrable functions. We shall say that a quadrature rule with nodes $x_{1}, \ldots, x_{n} \in[a, b]$ and positive weights $w_{1}, \ldots, w_{n}$ discretizes a collection of square integrable functions 
$f_{1}, \ldots, f_{m}$ defined on the interval $[a, b]$ if

$$
\int_{a}^{b} f_{i}(x) f_{j}(x) d x=\sum_{l=1}^{n} f_{i}\left(x_{l}\right) f_{j}\left(x_{l}\right) w_{l}
$$

holds for all $i=1, \ldots, m$ and $j=1, \ldots, m$.

If $x_{1}, \ldots, x_{n}, w_{1}, \ldots, w_{n}$ is a quadrature discretizing a collection of functions $f_{1}, \ldots, f_{m}$ in $L^{2}([a, b])$, then the map $T$ from the span $S$ of the $f_{j}$ to the Euclidean space $\mathbb{R}^{n}$ taking the function $f$ to the vector

$$
\left(\begin{array}{c}
f\left(x_{1}\right) \sqrt{w_{1}} \\
f\left(x_{2}\right) \sqrt{w_{2}} \\
\vdots \\
f\left(x_{n}\right) \sqrt{w_{n}}
\end{array}\right)
$$

is a Hilbert space isomorphism of the subspace $S$ onto the subspace of the Euclidean space $\mathbb{R}^{n}$ spanned by the vectors

$$
\left(\begin{array}{c}
f_{1}\left(x_{1}\right) \sqrt{w_{1}} \\
f_{1}\left(x_{2}\right) \sqrt{w_{2}} \\
\vdots \\
f_{1}\left(x_{n}\right) \sqrt{w_{n}}
\end{array}\right), \ldots,\left(\begin{array}{c}
f_{m}\left(x_{1}\right) \sqrt{w_{1}} \\
f_{m}\left(x_{2}\right) \sqrt{w_{2}} \\
\vdots \\
f_{m}\left(x_{n}\right) \sqrt{w_{n}}
\end{array}\right)
$$

2.2. Numerical rank. The numerical rank of a matrix $A$ to precision $\epsilon$ is defined to be the least integer $k$ such that $\sigma_{k+1}(A) \leq \epsilon$. Moreover, we define the numerical rank to precision $\epsilon$ of a collection $f_{1}, \ldots, f_{m}$ of square integrable functions on the interval $[a, b]$ to be the numerical rank to precision $\epsilon$ of the matrix

$$
\left(\begin{array}{cccc}
f_{1}\left(x_{1}\right) \sqrt{w_{1}} & f_{2}\left(x_{1}\right) \sqrt{w_{1}} & \ldots & f_{m}\left(x_{1}\right) \sqrt{w_{1}} \\
f_{1}\left(x_{2}\right) \sqrt{w_{2}} & f_{2}\left(x_{2}\right) \sqrt{w_{2}} & \ldots & f_{m}\left(x_{2}\right) \sqrt{w_{2}} \\
\ldots & \vdots & \ddots & \vdots \\
f_{1}\left(x_{n}\right) \sqrt{w_{n}} & f_{2}\left(x_{n}\right) \sqrt{w_{n}} & \ldots & f_{m}\left(x_{n}\right) \sqrt{w_{n}}
\end{array}\right)
$$

where $x_{1}, \ldots, x_{n}, w_{1}, \ldots, w_{n}$ is any quadrature discretizing $f_{1}, \ldots, f_{m}$.

2.3. Rank-revealing QR decompositions. A partial QR decomposition for an $m \times n$ matrix $A$ with $m \geq n$ is a factorization of the form

$$
A \Pi=Q\left(\begin{array}{cc}
R_{11} & R_{12} \\
0 & R_{22}
\end{array}\right),
$$

where $Q$ is an $m \times m$ orthogonal matrix, $\Pi$ is an $n \times n$ permutation matrix, $R_{11}$ is a $k \times k$ upper triangular matrix with nonnegative diagonal entries, $R_{12}$ is a $k \times(n-k)$ matrix, and $R_{22}$ is an $(m-k) \times(n-k)$ matrix. For any factorization of this type, we have

$$
\sigma_{i}\left(R_{11}\right) \leq \sigma_{i}(A) \quad \text { and } \quad \sigma_{j}\left(R_{22}\right) \geq \sigma_{k+j}(A)
$$

for $1 \leq i \leq k$ and $1 \leq j \leq(n-k)$. In [8], it is shown that for any $m \times n$ matrix $A$ with $m \geq n$ and any integer $1 \leq k \leq n$, there exists a factorization of this form such that $\left\|R_{11}^{-1} R_{12}\right\|_{\infty} \leq d(k, n)$,

$$
\sigma_{i}(A) \leq c(k, n) \sigma_{i}\left(R_{11}\right)
$$

holds for $i=1, \ldots, k$, and

$$
c(k, n) \sigma_{k+j}(A) \geq \sigma_{j}\left(R_{22}\right)
$$

holds for $j=1, \ldots, n-k$, where $c(k, n)=\sqrt{1+k(n-k)}$ and $d(k, n)=1$. Moreover, [8] gives a stable algorithm for the computation of partial QR factorizations satisfying bounds of this type with $c=\sqrt{1+n k(n-k)}$ and $d=\sqrt{n}$ which requires $O\left(m n^{2}\right)$ floating point operations - that is, the same asymptotic complexity as the well-known pivoted Gram-Schmidt algorithm².

\footnotetext{
${ }^{2}$ In fact, the performance of the scheme described in [8] depends on a parameter which affects both its running time and the resulting bounds. The values reported here represent but one possible configuration.
} 
Remark 2.1. Our implementation of the algorithm described in this paper uses the pivoted GramSchmidt procedure with reorthogonalization in place of the algorithm of [8] to compute rank-revealing QR factorizations. As is observed in [8] and discussed thoroughly in the monograph [2], the pivoted Gram-Schmidt algorithm with reorthogonalization, which is easier to implement and somewhat faster than the algorithm of [8], works well in practice despite the existence of counterexamples which show that it can fail in certain circumstances.

2.4. Generalized Chebyshev quadratures. A quadrature formula will be referred to as a Chebyshev quadrature for a set of $2 n$ linearly independent functions $\phi_{1}, \ldots, \phi_{2 n}:[a, b] \rightarrow \mathbb{R}$ if it consists of $2 n$ nodes and $2 n$ weights and integrates $\phi_{i}$, for all $i=1, \ldots, 2 n$.

The construction of a Chebyshev quadrature for an orthonormal collection of square integrable functions $u_{1}, \ldots, u_{k}$ defined on an interval $[a, b]$ is trivial given a preexisting quadrature

$$
x_{1}, \ldots, x_{n}, w_{1}, \ldots, w_{n}
$$

integrating products of those functions. Let

$$
U=\left(\begin{array}{cccc}
u_{1}\left(x_{1}\right) \sqrt{w_{1}} & u_{1}\left(x_{2}\right) \sqrt{w_{2}} & \cdots & u_{1}\left(x_{n}\right) \sqrt{w_{n}} \\
u_{2}\left(x_{1}\right) \sqrt{w_{1}} & u_{2}\left(x_{2}\right) \sqrt{w_{2}} & \cdots & u_{2}\left(x_{n}\right) \sqrt{w_{n}} \\
\vdots & & & \vdots \\
u_{k}\left(x_{1}\right) \sqrt{w_{1}} & u_{k}\left(x_{2}\right) \sqrt{w_{2}} & \cdots & u_{k}\left(x_{n}\right) \sqrt{w_{n}}
\end{array}\right)
$$

and

$$
r=\left(\begin{array}{c}
r_{1} \\
r_{2} \\
\vdots \\
r_{k}
\end{array}\right)
$$

where $r_{i}, i=1, \ldots, k$, is defined by

$$
r_{i}=\int_{a}^{b} u_{i}(x) d x .
$$

By virtue of the orthonormality of the $u_{1}, \ldots, u_{k}$ and the requirement that the quadrature integrates products of the $u_{i}$, the rows of the matrix $U$ are orthonormal. It follows that the matrix $U$ has $k$ nonzero singular values, all of which are 1. The results of [8] discussed in Section 2.3 now imply that a decomposition of $U$ of the form

$$
U \Pi=Q\left(\begin{array}{ll}
R_{11} & R_{12}
\end{array}\right),
$$

where $Q$ is an orthogonal $k \times k$ matrix, $R_{12}$ is an $k \times(n-k)$ matrix and $R_{11}$ is a $k \times k$ matrix such that

$$
\frac{1}{\sqrt{1+n k(n-k)}} \leq \sigma_{j}\left(R_{11}\right) \leq 1
$$

for $1 \leq j \leq k$, can be computed stably in at most $O\left(n^{3}\right)$ operations. A vector $z$ with at most $k$ nonzero entries such that

$$
U z=\left(\begin{array}{c}
r_{1} \\
r_{2} \\
\vdots \\
r_{k}
\end{array}\right)
$$

can be computed by solving the $k \times k$ linear system

$$
R_{11} \hat{z}=Q^{*} r
$$

the condition number of which is bounded by

$$
\sqrt{1+n k(n-k)}
$$


by virtue of the inequality (2.3), and setting $z$ to be

$$
\Pi^{-1}\left(\begin{array}{c}
\tilde{z} \\
0 \\
\vdots \\
0
\end{array}\right) .
$$

If $i_{1}, \ldots, i_{k}$ denote the indices of the nonzero components of $z$ and we let

$$
y_{j}=x_{i_{j}} \quad \text { and } \quad v_{j}=z_{i_{j}} \sqrt{w_{i_{j}}}
$$

for $j=1, \ldots, k$, then we have

$$
\left(\begin{array}{cccc}
u_{1}\left(y_{1}\right) & u_{1}\left(y_{2}\right) & \cdots & u_{1}\left(y_{k}\right) \\
u_{2}\left(y_{1}\right) & u_{2}\left(y_{2}\right) & \cdots & u_{2}\left(y_{k}\right) \\
\vdots & & \cdots & \vdots \\
u_{k}\left(y_{1}\right) & u_{k}\left(y_{2}\right) & \cdots & u_{k}\left(y_{k}\right)
\end{array}\right)\left(\begin{array}{c}
v_{1} \\
v_{2} \\
\vdots \\
v_{k}
\end{array}\right)=r
$$

that is, $y_{1}, \ldots, y_{k}, v_{1}, \ldots, v_{k}$ is a Chebyshev quadrature for $u_{1}, \ldots, u_{k}$.

2.5. Chebyshev quadratures and interpolation. If $f$ is a linear combination

$$
f(x)=\sum_{j=1}^{k} \alpha_{j} u_{j}(x)
$$

of the orthonormal functions $u_{1}, \ldots, u_{k}$ in $L^{2}[a, b]$ and $x_{1}, \ldots, x_{n}, w_{1}, \ldots, w_{n}$ is a quadrature integrating products of the the $u_{j}$, then the coefficients $\alpha_{1}, \ldots, \alpha_{k}$ in the expansion (2.5) can be computed stably from the values of the function $f$ at the quadrature nodes $x_{1}, \ldots, x_{n}$. In particular, let $U$ be the $n \times k$ matrix whose entries are given by

$$
U_{i j}=u_{j}\left(x_{i}\right) \sqrt{w_{i}}
$$

let

$$
F=\left(\begin{array}{c}
f\left(x_{1}\right) \\
f\left(x_{2}\right) \\
\vdots \\
f\left(x_{n}\right)
\end{array}\right)
$$

and denote by $W$ the $n \times n$ diagonal matrix with entries

$$
W_{i i}=\sqrt{w_{i i}}
$$

Then

$$
\left(\begin{array}{c}
\alpha_{1} \\
\alpha_{2} \\
\vdots \\
\alpha_{k}
\end{array}\right)=U^{*} W F .
$$

Note that $U U^{*}(W F)=W F$ since $W F$ is in the span of the column space of $\mathrm{U}$ by assumption. The computation of $\alpha_{1}, \ldots, \alpha_{k}$ in this fashion is entirely numerically stable since the rows of $U^{*}$ are orthonormal and $W$ is a diagonal matrix. If a scheme is available for evaluating the functions $u_{j}$ at arbitrary points $x$, then this mechanism provides a stable means for interpolating functions in the span of $u_{1}, \ldots, u_{k}$ from $x_{1}, \ldots, x_{n}$ to arbitrary points.

In fact, interpolation from the nodes of a Chebyshev quadrature for $u_{1}, \ldots, u_{k}$ constructed in the manner described in the preceding section is also stable. To see this, suppose $x_{i_{1}}, \ldots, x_{i_{k}}$ are the nodes 
of such a quadrature. Then, by virtue of the inequality (2.3) appearing in the preceding section, the condition number of the matrix $\tilde{U}$ defined by

$$
\tilde{U}=\left(\begin{array}{cccc}
u_{1}\left(x_{i_{1}}\right) \sqrt{w_{i_{1}}} & u_{2}\left(x_{i_{1}}\right) \sqrt{w_{i_{1}}} & \cdots & u_{k}\left(x_{i_{1}}\right) \sqrt{w_{i_{1}}} \\
u_{1}\left(x_{i_{2}}\right) \sqrt{w_{i_{2}}} & u_{2}\left(x_{i_{2}}\right) \sqrt{w_{i_{2}}} & \cdots & u_{k}\left(x_{i_{2}}\right) \sqrt{w_{i_{2}}} \\
\vdots & & \cdots & \vdots \\
u_{1}\left(x_{i_{k}}\right) \sqrt{w_{i_{k}}} & u_{2}\left(x_{i_{k}}\right) \sqrt{w_{i_{k}}} & \cdots & u_{k}\left(x_{i_{k}}\right) \sqrt{w_{i_{k}}}
\end{array}\right)
$$

is bounded by $\sqrt{1+n k(n-k)}$. By construction, the vector

$$
\left(\begin{array}{c}
f\left(x_{i_{1}}\right) \sqrt{w_{i_{1}}} \\
f\left(x_{i_{2}}\right) \sqrt{w_{i_{2}}} \\
\vdots \\
f\left(x_{i_{k}}\right) \sqrt{w_{i_{k}}}
\end{array}\right)
$$

is in the span of the columns of $\tilde{U}$, so it follows that the linear system

$$
\tilde{U}\left(\begin{array}{c}
\alpha_{1} \\
\alpha_{2} \\
\vdots \\
\alpha_{k}
\end{array}\right)=\left(\begin{array}{c}
f\left(x_{i_{1}}\right) \sqrt{w_{i_{1}}} \\
f\left(x_{i_{2}}\right) \sqrt{w_{i_{2}}} \\
\vdots \\
f\left(x_{i_{k}}\right) \sqrt{w_{i_{k}}}
\end{array}\right)
$$

admits a unique solution which can be stably computed.

\section{NYSTRÖM DISCRETIZATION}

The Nyström discretization of the integral equation

$$
\lambda \sigma(x)+\int_{\Gamma} K(x, y) \sigma(y) d S(y)=u(x)
$$

operates by fixing local representations for solutions $\sigma$ of the form

$$
\sigma(y) \approx \sum_{j=1}^{N} \alpha_{j} \phi_{j}(y)
$$

and approximating the integral in Equation (3.1) via quadrature formulae integrating functions of the form

$$
\sum_{j=1}^{N} K(x, y) \alpha_{j} \phi_{j}(y)
$$

Typically, more than one quadrature formula of this type is required, with each integrating functions of the form (3.2) for various values or ranges of values of $x$. See [1] for a detailed discussion of the Nyström discretization of boundary integral equations.

We now describe a very general Nyström framework for the discretization of Laplace and Helmholtz boundary integral equations of the form (3.1). We begin by assuming that $\Gamma$ is divided into $n$ curve segments, $\Gamma_{1}, \ldots, \Gamma_{n}$, not necessarily of equal length. For each curve segment $\Gamma_{j}$ we will require the following:

(1) An orthonormal collection of $k$ basis functions $\phi_{1}, \ldots, \phi_{k}$ in $L^{2}\left(\Gamma_{j}\right)$,

(2) A scheme for interpolating the basis functions $\phi_{1}, \ldots, \phi_{k}$ from nodes $\lambda_{1}, \ldots, \lambda_{k}$ to their values at arbitrary points,

(3) A "far" quadrature formula exact for integrals of the form

$$
\int_{\Gamma_{j}} K(x, y) \sigma_{i}(y) d S(y)
$$

$i=1, \ldots, k$, when $x$ sufficiently distant from $\Gamma_{j}$, 
(4) A "near" quadrature formula exact for integrals of the form

$$
\int_{\Gamma_{j}} K(x, y) \sigma_{i}(y) d S(y)
$$

$i=1, \ldots, k$, when $x$ is outside of, but close to, $\Gamma_{j}$,

(5) A set of $k$ "diagonal" quadrature formulas each of the form

$$
\int_{\Gamma_{j}} K\left(\lambda_{i}, y\right) \sigma(y) d S(y) \approx \sum_{l=1}^{M_{i}} K\left(\lambda_{i}, y_{l}\right) \sigma\left(y_{l}\right) w_{l}
$$

exact whenever $\sigma$ is in the span of $\sigma_{1}, \ldots, \sigma_{k}$.

The method proceeds under the assumption that the restriction of the unknown solution $\sigma$ in equation (3.1) to the curve segment $\Gamma_{j}$ can be represented as linear combinations of the basis functions $\phi_{1}, \ldots, \phi_{k}$ for $\Gamma_{j}$. Let $\Gamma_{j}$ and $\Gamma_{i}$ be two curve segments, not necessarily distinct. We will denote by $\phi_{1}, \ldots, \phi_{n}$ the basis functions on the curve segment $\Gamma_{j}$; by $s_{1}, \ldots, s_{n}$ the interpolation nodes on the curve segment $\Gamma_{j}$; and by $t_{1}, \ldots, t_{m}$ the interpolation nodes on the curve segment $\Gamma_{i}$. The integral equation

$$
T_{i j} \sigma(x)=u(x),
$$

where $T_{i j}$ is the integral operator mapping functions on $\Gamma_{j}$ to functions on $\Gamma_{i}$ defined by

$$
T_{i j} \sigma(x)=\int_{\Gamma_{j}} K(x, y) \sigma(y) d y
$$

is then discretized by repeating the following sequence of steps for each interpolation node $t$ on $\Gamma_{i}$ :

(1) The appropriate quadrature formula $x_{1}, \ldots, x_{l}, w_{1}, \ldots, w_{l}$ for functions of the form

$$
K(t, s) \sigma_{u}(s), \quad u=1, \ldots, n,
$$

is determined. That is, depending on the location of $t$ relative to the curve segment $\Gamma_{j}$, either the "far" quadrature rule, the "near" quadrature rule, or one of the diagonal quadrature rules is selected.

(2) The kernel $K(x, y)$ is evaluated at the points $\left(t, x_{r}\right)$ for $r=1, \ldots, l$ and the $1 \times l$ vector $v$ with entries

is formed.

$$
v_{r}=K\left(t, x_{r}\right) w_{r}
$$

(3) The $1 \times l$ vector $v$ is multiplied on the right by the $l \times n$ matrix interpolating the basis functions $\phi_{1}, \ldots, \phi_{n}$ from the interpolation nodes $s_{1}, \ldots, s_{n}$ on $\Gamma_{j}$ to the quadrature nodes $x_{1}, \ldots, x_{l}$.

(4) The entries $\left\{\alpha_{s}\right\}$ of the resulting $1 \times n$ vector give a single linear equation

$$
\alpha_{1} \sigma\left(s_{1}\right)+\alpha_{2} \sigma\left(s_{2}\right)+\ldots \alpha_{n} \sigma\left(s_{n}\right)=u(t)
$$

constraining the values of the solution $\sigma$ at the nodes $s_{1}, \ldots, s_{n}$.

The result of repeating this procedure for each of the $m$ interpolation nodes is an $m \times n$ linear system of the form

$$
\left(\begin{array}{cccc}
a_{11} & a_{12} & \ldots & a_{1 n} \\
a_{21} & a_{22} & \ldots & a_{2 n} \\
\vdots & \vdots & \ddots & \vdots \\
a_{m 1} & a_{m 2} & \ldots & a_{m n}
\end{array}\right)\left(\begin{array}{c}
\sigma\left(s_{1}\right) \\
\sigma\left(s_{2}\right) \\
\vdots \\
\sigma\left(s_{n}\right)
\end{array}\right)=\left(\begin{array}{c}
u\left(t_{1}\right) \\
u\left(t_{2}\right) \\
\vdots \\
u\left(t_{m}\right)
\end{array}\right)
$$

discretizing the integral equation

$$
T_{i j} \sigma=u \text {. }
$$

Repeating the above procedure for each pair of curve segments $\Gamma_{j}$ and $\Gamma_{i}$, and accounting for the constant term in equation (3.1) results in a discrete system of $N$ equations in $N$ unknowns of the form

$$
\lambda x+A x=y,
$$

where $N$ is equal to the sum of the number of interpolation nodes $n_{j}$ on each curve segment $\Gamma_{j}$ over $j=1, \ldots, n$ and $A$ is a matrix formed by concatenating the discrete matrices representing the $T_{i j}$. 
Solving the amalgamated system yields the values of the unknown function $\sigma$ in (3.1) at the interpolation nodes of each of the curve segments $\Gamma_{j}$. The value of $\sigma$ at any point $x$ on $\Gamma$ can then be computed in $O(1)$ operations using the appropriate interpolation formula. Moreover, the value of a layer potential

$$
u(x)=\int_{\Gamma} K(x, y) \sigma(y) d y
$$

can be computed for any $x$ sufficiently far enough away from the curve $\Gamma$ in $O(n)$ operation using the far quadrature formulas for the curve segments $\Gamma_{j}, j=1, \ldots, n$. For points close to the curve, an adaptive Gaussian quadrature scheme which relies on the ability to evaluate the charge distribution at any point via interpolation can be used to compute the value of the layer potential.

\section{Charge bases}

In this section, we show that under very mild assumptions, a small finite orthonormal basis spanning the restrictions of solutions of a Laplace or Helmholtz boundary integral equation on a contour $\Gamma$ to a small curve segment $\Gamma_{0} \subset \Gamma$ can be constructed. We will refer to such a basis as a "charge basis" for the contour $\Gamma_{0}$ (the underlying integral equation will always be apparent from context).

In the interests of brevity, we will restrict our attention here to the boundary integral equation

$$
-\frac{1}{2} \sigma(x)+\int_{\Gamma}\left(\frac{i}{4} \nu(y) \cdot \nabla_{y}+1\right) H_{0}(k|x-y|) \sigma(y) d S(y)=u(x),
$$

where $H_{0}$ is the Hankel function of zeroth order and $\nu(y)$ denotes the outward unit normal vector on the contour $\Gamma$. The equation (4.1) is typically used to obtain a solution of the exterior Dirichlet boundary value problem for the Helmholtz equation with wavenumber $k$ (see, for instance, [5]). The argument given here clearly applies to a variety of other boundary integral equations with minor modifications.

4.1. Partial wave expansions. If the function $\phi: \mathbb{R}^{2} \rightarrow \mathbb{R}$ satisfies the Helmholtz equation

$$
\left(\nabla^{2}+k^{2}\right) \phi=0
$$

in a disc $D$ of radius $R>0$ centered at $x_{0}$ and the radiation condition

$$
\lim _{t \rightarrow \infty} \phi(t x) e^{-i k t \cdot|x|}=O\left(t^{-1 / 2}\right)
$$

uniformly at infinity, then $\phi$ can be represented uniquely as a $J$-expansion in $D$; i.e., in the form

$$
\phi(x)=\sum_{m=-\infty}^{\infty} \alpha_{m} J_{m}(k r) e^{i m \theta},
$$

where $(r, \theta)$ denote the usual polar coordinates centered at $x_{0}$ and $J_{m}$ is the Bessel function of the first kind of order $m$. The following theorem, which appears in [10], establishes the convergence rate of the expansion (4.2).

THEOREM 4.1. If $D_{1} \subset D$ is a disc of radius $R_{1}<R$ centered at $x_{0}$, then there exists a constant $c>0$ such that for $x \in D_{1}$ and $N>|k| R_{1}$,

$$
\left|\phi(x)-\sum_{m=-N}^{N} \alpha_{m} J_{m}(k r) e^{i m \theta}\right|<c\left(\frac{R_{1}}{R}\right)^{N} .
$$

4.2. Rank of interaction. If $\Gamma_{0}$ and $\Gamma_{1}$ are two disjoint compact connected Lipschitz curves in the plane and $K(x, y)$ is one of the potential theoretic kernels considered in this paper, then the mapping defined by

$$
T f(x)=\int_{\Gamma_{1}} K(x, y) f(y) d S(y)
$$

is compact as an operator $L^{2}\left(\Gamma_{0}\right) \rightarrow L^{2}\left(\Gamma_{1}\right)$. We shall define the rank of interaction of the curve segments $\Gamma_{0}$ and $\Gamma_{1}$ under the kernel $K$ as the least integer $m$ such that either $m-1$ singular values of $T$ are less than the prescribed precision $\epsilon$. 


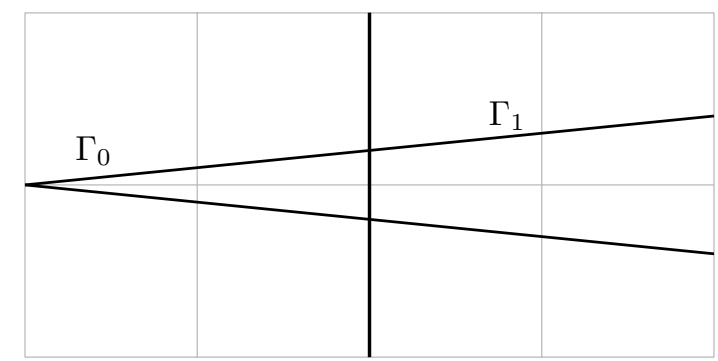

Figure 1: The rank of interaction of the two curve segments $\Gamma_{0}$ and $\Gamma_{1}$ under the potential theoretic kernels considered in this paper is low regardless of the angle of the corner.

If the rank of interaction of the operator (4.3) is $m$ to precision $\epsilon$, then there exists a quadrature formula of the form

$$
\int_{\Gamma_{1}} K(x, y) f(y) d S(y) \approx \sum_{j=1}^{m} K\left(x, y_{j}\right) f\left(y_{j}\right) w_{j}
$$

with the $y_{j}$ lying in $\Gamma_{1}$, which holds with precision approximately $\epsilon$ for $x \in \Gamma_{0}$ (note that the kernels considered in this paper are bounded almost everywhere for $x \in \Gamma_{0}$ and $\left.y \in \Gamma_{1}\right)$. A discussion of the existence and numerical computation of such quadratures can be found in [4].

Although the rank of interaction between two curve segments under one of the potential-theoretic kernels considered here can be high, for most curves appearing in practice the singular values of the integral operators corresponding to interactions between neighboring curve segments decay exponentially (see, for instance, [6], which contains several relevant examples). In the particular case of corner regions, which are the focus of this paper, the rank of interaction between a neighborhood of a corner and the neighboring portions of the curve is typically low regardless of the angle of the corner. The situation is illustrated in Figure 1.

4.3. Construction of charge bases. In what follows, we shall fix a simply-connected domain $\Omega$ in the plane whose boundary $\Gamma$ is a compact, connected Lipschitz curve. Our aim is to produce an orthonormal basis approximately spanning the set of solutions of the boundary integral equation (4.1) to a small curve segment $\Gamma_{0} \subset \Gamma$. We will denote by $K(x, y)$ the integral kernel

$$
\left(\frac{i}{4} \nu(y) \cdot \nabla_{y}+1\right) H_{0}(k|x-y|)
$$

appearing in equation (4.1). Moreover, will shall let $B$ be a disc of minimum radius which contains $\Gamma_{0}$ and we will denote by $\Gamma_{1}$ the portion of the contour $\Gamma$ contained in $2 B \backslash B$. Finally, we will let $\Gamma_{2}$ denote the portion of the curve $\Gamma$ contained in the complement of the ball $2 B$. Figure 2 depicts the situation.

The boundary integral equation (4.1) can be rearranged as

$$
\begin{aligned}
-\frac{1}{2} \sigma(x)+ & \int_{\Gamma_{0}} K(x, y) \sigma(y) d S(y)=u(x)- \\
& \int_{\Gamma_{2}} K(x, y) \sigma(y) d S(y)-\int_{\Gamma_{1}} K(x, y) \sigma(y) d S(y) .
\end{aligned}
$$

Under the assumption that the right hand side $u(x)$ satisfies the Helmholtz equation in the disc $2 B$, we can, by virtue of Theorem 4.1, introduce the approximation

$$
u(x)+\int_{\Gamma_{2}} K(x, y) \sigma(y) d S(y) \approx \sum_{m=-N}^{N} \alpha_{m} J_{m}(k r) e^{i m \theta},
$$




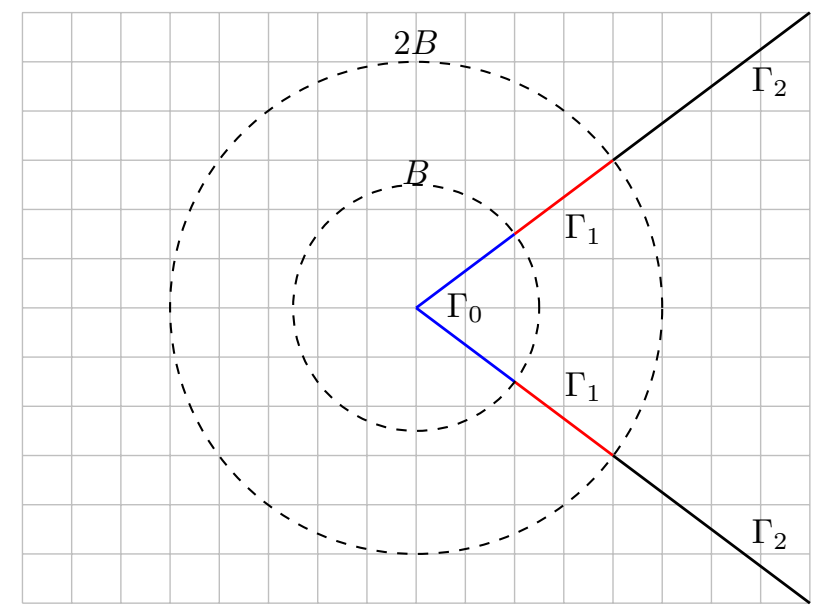

Figure 2

which holds for $x \in \Gamma_{0}$. Moreover, as described in Section 4.2, when $x \in \Gamma_{0}$ the third term on the right hand side of equation (4.4) can be approximated as

$$
\int_{\Gamma_{1}} K(x, y) f(y) d S(y) \approx \sum_{j=1}^{M} K\left(x, y_{j}\right) f\left(y_{j}\right) w_{j}
$$

where the $y_{j}$ lie in $\Gamma_{1}$ and $M$ is the rank of interaction of $\Gamma_{0}$ with $\Gamma_{1}$. It follows that the restriction of $\sigma$ to the curve segment $\Gamma_{0}$ satisfies the integral equation

$$
-\frac{1}{2} \sigma(x)+\int_{\Gamma_{0}} K(x, y) \sigma(y) d S(y)=\sum_{m=-N}^{N} \alpha_{m} J_{m}(k r) e^{i m \theta}+\sum_{j=1}^{M} K\left(x, y_{j}\right) f\left(y_{j}\right) w_{j} .
$$

It is now clear how to form a charge basis for $\Gamma_{0}$. We observe that the restriction of (4.1) to $\Gamma_{0}$ is invertible as an operator $L^{2}\left(\Gamma_{0}\right) \rightarrow L^{2}\left(\Gamma_{0}\right)$ and form the collection of functions obtained by solving the restricted integral equation for each of the functions of the form

$$
J_{m}(k r) e^{i m \theta} \quad \text { and } \quad K\left(x, y_{j}\right)
$$

appearing in (4.6). The resulting functions are orthonormalized in order to form a charge basis.

4.4. Convergence estimate. In this section, we derive a crude estimate on the number of charge basis functions required to achieve a given $L^{\infty}\left(\Gamma_{0}\right)$ precision $\epsilon$ for the approximation (4.6). And of course, this quantity is related to the $L^{2}\left(\Gamma_{0}\right)$ error in the approximation of restricted solutions by the charge basis through the $L^{2}\left(\Gamma_{0}\right)$ norm of the inverse of the restriction of the integral operator (4.1) and the arclength of the curve $\Gamma_{0}$.

In particular, if $N$ is chosen so that

$$
N \geq \max \left(|k| R_{1}, \frac{-\log (\epsilon)+\log (c)}{\log (2)}\right),
$$

where $R_{1}$ is the radius of $B$ and $c$ is the constant appearing in Theorem 4.1, then the approximation (4.5) is guaranteed to achieve precision $\epsilon$. The rank of interaction $M$ of $\Gamma_{0}$ and $\Gamma_{1}$ depends on the geometry of the contour $\Gamma$ and the wavenumber $k$; however, in typical cases - especially when $\Gamma_{0}$ is taken to be a small region around a corner point $-M$ behaves in a similar fashion.

Thus the number of charge basis functions required for precision $\epsilon$ is roughly

$$
\max \left(|k| R_{1}, O(-\log (\epsilon))\right) .
$$

In other words, the convergence of approximations with respect to charge bases is exponential once a certain number of "terms per wavelength" has been achieved. This means that the number of basis 
functions necessary to represent a charge distribution on many types of complicated curve segments (e.g. corner regions) is comparable with the number required to represent a charge distribution on a smooth curve segment using typical approaches (e.g., piecewise Gaussian polynomials). This estimate is consistent with our numerical experiments, which are presented in Section 7.

Remark 4.1. This estimate only provides a very crude upper bound for the dimension of the resulting charge basis; charge bases are almost always much smaller than this estimate suggests. Moreover, in practice, the number of charge basis functions seems to have only a weak dependence on the rank of interaction of $\Gamma_{0}$ and $\Gamma_{1}$.

Remark 4.2. In the case of the boundary integrals related to Laplace's equation, considerations involving the wavenumber are (obviously) unnecessary. For those integral equations, in typical cases the number of charge basis functions behaves as $O(-\log (\epsilon))$.

\section{Purpose-made quadratures}

In this section, we describe an algorithm for the construction of a charge basis $\sigma_{1}, \ldots, \sigma_{k}$ for a Laplace or Helmholtz boundary integral equation

$$
\lambda \sigma(x)+\int_{\Gamma} K(x, y) \sigma(y) d S(y)=u(x)
$$

over a corner curve segment $\Gamma_{0} \subset \Gamma$ as well as an algorithm for the computation of purpose-made quadratures for its discretization over $\Gamma_{0}$ given a charge basis for $\Gamma_{0}$.

5.1. Construction of a charge basis. The algorithm of this section takes as input a precision $\epsilon$, a parameterization $r:[-1,1] \rightarrow \Gamma_{0}$ that maps 0 to the corner point of $\Gamma_{0}$, and two integer parameters $s$ and $k$ whose roles will be described below. It proceeds as follows:

1. Form a simply-graded mesh ${ }^{1}$ on the corner curve segment $\Gamma_{0}$ by constructing the subintervals of $[-1,1]$ with endpoints

$$
\frac{1}{2^{j}} \text { and }-\frac{1}{2^{j}} \text { for } j=1,2, \ldots, s,
$$

and taking the image of the resulting subintervals under the parameterization $r$.

2. Discretize the restricted boundary integral equation

$$
\lambda \sigma(x)+\int_{\Gamma_{0}} K(x, y) \sigma(y) d S(y)=u(x) \quad\left(x \in \Gamma_{0}\right)
$$

via the Nyström method under the assumption that the solution $\sigma$ can be represented as a $k$ th order piecewise Legendre expansion over the simply-graded mesh formed in Step 1. Denote by $x_{1}, \ldots, x_{m}, w_{1}, \ldots, w_{m}$ the nodes and weights of the piecewise Legendre quadrature which represents solutions of $(5.2)$ on $\Gamma_{0}$.

3. Solve the discrete linear system formed in Step 2 for a collection of right-hand-sides consisting of multipoles and functions of the form

$$
K\left(x, y_{j}\right),
$$

where the $y_{j}$ are points on $\Gamma$ adjacent to $\Gamma_{0}$. The order of the multipole functions is chosen so as to ensure very high accuracy in the approximation of potential functions on $\Gamma_{0}$. Similarly, the points $y_{j}$ adjacent to $\Gamma_{0}$ are chosen to be the nodes of a quadrature which discretizes the operator $T: L^{2}\left(\Gamma_{0}\right) \rightarrow L^{2}\left(\Gamma_{1}\right)$ given by

$$
T f(x)=\int_{\Gamma_{0}} K(x, y) f(y) d y
$$

to high accuracy.

Denote the resulting functions by $u_{1}, \ldots, u_{n}$. Note that the values of the $u_{j}$ at arbitrary points on $\Gamma_{0}$ can be calculated using standard interpolation techniques for piecewise Legendre polynomials.

\footnotetext{
${ }^{1}$ The terminology is adapted from [9]. Also see [4].
} 
4. Form the $m \times n$ matrix $A$ whose entries are given by

$$
A_{i j}=u_{j}\left(x_{i}\right) \sqrt{w_{i}} .
$$

5. Form a rank-revealing QR decomposition

$$
A \Pi=Q\left(\begin{array}{cc}
R_{11} & R_{12} \\
0 & R_{22}
\end{array}\right)
$$

for $A$ and let $k$ be the least integer such that the $k$ th singular value of $A$ is less than the input precision $\epsilon$.

6. Define the charge basis functions $\sigma_{1}, \ldots, \sigma_{k}$ via the formula

$$
\sigma_{j}\left(x_{i}\right)=Q_{i j} / \sqrt{w_{i}},
$$

where $Q_{i j}$ refers to the $i j$ th entry of the matrix $Q$ formed in the preceding step. Note that since the values of the $\sigma_{j}$ are known at the points of a piecewise Legendre quadrature, their values at any point on $\Gamma_{0}$ can be readily obtained via interpolation.

The parameters $s$ and $k$ should be chosen so as to ensure that the piecewise Legendre quadrature used to discretize solutions of the restricted boundary integral equation (5.2) integrates products of the solutions obtained in Step 3.

Remark 5.1. Simply-graded meshes are not necessarily sufficient to represent solutions of the boundary integral equations to double precision, at least if the computations are performed using double precision arithmetic. However, the precomputations described in this section may be performed in extended precision (FORTRAN REAL*16) arithmetic if necessary to achieve higher accuracy.

5.2. Construction of the quadrature formulae. In this section, we detail the construction of the quadrature formulae for the discretization of the boundary integral equation (5.1) over $\Gamma_{0}$.

The algorithm of this section takes as input the quadrature $x_{1}, \ldots, x_{m}, w_{1}, \ldots, w_{m}$ and the charge basis $\sigma_{1}, \ldots, \sigma_{k}$ formed using the procedure of the preceding section as well as an integer parameter $k_{\text {lege }}$. We may, by virtue of the parameterization $r:[-1,1] \rightarrow \Gamma_{0}$ regard the functions $\sigma_{j}$ as given over the interval $[-1,1]$.

The algorithm proceeds as follows:

1. Construct a Chebyshev quadrature for the charge basis functions $\sigma_{1}, \ldots, \sigma_{k}$. The nodes $\lambda_{1}, \ldots, \lambda_{k}$ of this quadrature serve as discretization nodes for Nyström procedure of Section 3. Note that the nodes $\lambda_{1}, \ldots, \lambda_{k}$ are guaranteed to be stable interpolation nodes for the charge basis functions (as described in Section 2.5).

2. Form the "far" quadrature required by the Nyström procedure by constructing a Chebyshev quadrature for functions of the form

$$
\sigma_{j}(x)(p(x)+q(x)),
$$

where $1 \leq j \leq k, p(x)$ is a polynomial of degree $k_{\text {lege }}$ on the interval $[-1,0]$, and $q(x)$ is a polynomial of degree $k_{\text {lege }}$ on the interval $[0,1]$.

3. Construct the "near" quadrature by constructing a Chebyshev quadrature for functions of the form

$$
\log |t-x| \sigma_{j}(x),
$$

where $j=1, \ldots, k$ and $t$ is a point on $\Gamma$ close to $\Gamma_{0}$.

4. For each $i=1, \ldots, k$, construct a diagonal quadrature for functions of the form

$$
K\left(\lambda_{i}, x\right) \sigma_{j}(x),
$$

$j=1, \ldots, k$.

5. For each of the $k+2$ quadratures constructed in the last section, form the matrix interpolating the charge basis functions from the discretization nodes $\lambda_{1}, \ldots, \lambda_{k}$ to the quadrature nodes. This can be achieved via the procedure described in Section 2.5. 


\section{UNIVERSAL QUADRATURES FOR DOMAINS WITH CORNERS}

We now indicate how the procedure of the preceding section can be modified in order to produce a collection of quadrature formulae suitable for the discretization of a very general class of domains with corner points.

We shall say that a contour $\Gamma$ is a corner region with corner point at $\left(x_{0}, y_{0}\right)$ if it admits a parameterization $r(t)=(x(t), y(t))$ such that

$$
\begin{aligned}
& x(t)= \begin{cases}x_{0}+a_{1} t+a_{2} t^{2}+a_{3} t^{3}+\ldots & \text { for } a<t<0 \\
x_{0}+b_{1} t+b_{2} t^{2}+b_{3} t^{3}+\ldots & \text { for } 0<t<b\end{cases} \\
& y(t)= \begin{cases}y_{0}+c_{1} t+c_{2} t^{2}+c_{3} t^{3}+\ldots & \text { for } a<t<0 \\
y_{0}+d_{1} t+d_{2} t^{2}+d_{3} t^{3}+\ldots & \text { for } 0<t<b,\end{cases}
\end{aligned}
$$

where the series converge uniformly for $t \neq 0$, and $\left(a_{1}, c_{1}\right) \neq\left(b_{1}, d_{1}\right)$. We can assume without loss of generality that $(6.1)$ is an arclength parameterization; i.e., that $x^{\prime}(t)^{2}+y^{\prime}(t)^{2}=1$. For a contour of this type, we define the oriented angle $\theta$ of the corner as the angle of the counter-clockwise rotation required to transform the vector

$$
\lim _{t \rightarrow 0^{-}}\left(\begin{array}{l}
x^{\prime}(t) \\
y^{\prime}(t)
\end{array}\right)=\left(\begin{array}{l}
a_{1} \\
c_{1}
\end{array}\right)
$$

into the vector

$$
\lim _{t \rightarrow 0^{+}}\left(\begin{array}{c}
x^{\prime}(t) \\
y^{\prime}(t)
\end{array}\right)=\left(\begin{array}{l}
b_{1} \\
d_{1}
\end{array}\right) .
$$

Furthermore, we define the left and right instantaneous curvatures by

$$
\begin{aligned}
\kappa_{-} & =\lim _{t \rightarrow 0^{-}}\left[x^{\prime}(t) y^{\prime \prime}(t)-y^{\prime}(t) x^{\prime \prime}(t)\right] \\
& =2\left(a_{1} c_{2}-c_{1} a_{2}\right) \\
\kappa_{+} & =\lim _{t \rightarrow 0^{+}}\left[x^{\prime}(t) y^{\prime \prime}(t)-y^{\prime}(t) x^{\prime \prime}(t)\right] \\
& =2\left(b_{1} d_{2}-d_{1} b_{2}\right) .
\end{aligned}
$$

Given the angle $\theta$ and the two instantaneous curvatures $\kappa_{-}$and $\kappa_{+}$, the coefficients $a_{1}, a_{2}, b_{1}, b_{2}$, $c_{1}, c_{2}, d_{1}, d_{2}$ in the parameterization (6.1) can be recovered up to rotation and translation - that is, the coefficients corresponding to a contour which is the image of $\Gamma$ under translation and rotation can be readily derived from $\theta, \kappa_{-}$, and $\kappa+$. Note that this depends on our assumption that the parameterization is with respect to arclength (which reduces the number of free variables to 4).

It is an obvious consequence of the rotation and translation invariance of Laplace and Helmholtz problems that a charge basis for corner regions whose oriented angles and instantaneous curvatures lie in given ranges

$$
\begin{array}{r}
\theta \in\left[\theta_{1}, \theta_{2}\right] \\
\kappa_{-}, \kappa_{+} \in\left[\kappa_{1}, \kappa_{2}\right]
\end{array}
$$

and such that the terms of order greater than 2 in the parameterization (6.1) are 0 can be formed by sampling a large number of angles $\theta$ and curvatures $\kappa_{-}$and $\kappa_{+}$in the desired intervals and combining the charge bases corresponding to corner regions with each possible combination of these parameters. However, it is also possible to remove the restriction that the higher order terms in (6.1) be zero. To do so, we observe that a perturbation in the higher order terms in (6.1) results in a piecewise smooth perturbation of Laplace or Helmholtz integral kernels defined over the contour $\Gamma$. By artificially augmenting the basis obtained by combining charge bases for various values of $\theta, \kappa_{-}, \kappa_{+}$with functions of the form

$$
p(x)+q(x)
$$

where $p(x)$ is a polynomial over a given degree on the interval $[a, 0)$ and $q(x)$ is a polynomial over a given degree on the interval $(0, b]$, we obtain a basis which approximately spans the space of restrictions of a Laplace or Helmholtz boundary integral equation to corner contours of the form (6.1) for a wide 
class of corners with oriented angles $\theta \in\left[\theta_{1}, \theta_{2}\right]$ and instantaneous curvatures $\kappa_{-}, \kappa_{+} \in\left[\kappa_{1}, \kappa_{2}\right]$. In a similar manner, a collection of quadratures for the discretization of boundary integral equations over such regions - that is, the far, near, and diagonal regimes described in the preceding section - can be obtained by artificially augmenting the collections of functions which serve as input to the Chebyshev quadrature procedure with bases for piecewise smooth functions.

\section{Numerical RESUlts}

The algorithm of this paper for the construction of universal quadratures for domains with corners was implemented in Fortran 77 as was (the obvious) modification of that algorithm for the construction of purpose-made quadratures for specific curve segments. The resulting code was complied with the Lahey/Fujitsu Linux64 Fortran Compiler Release 8.10a. Timings were performed on a PC with an Intel Core i7 $2.67 \mathrm{GHz}$ processor and 12GB of memory and refer to wall clock time. No attempt was made to parallelize any of the code.

All boundary integral equations were inverted using a simple direct solver which is $O\left(n^{2}\right)$ in the number of discretization nodes $n$. The precise algorithm will be reported at a later date.

\subsection{Purpose-made quadratures.}

7.1.1. A Laplace Neumann problem. In this numerical experiment, we solved the exterior Neumann problem

$$
\begin{array}{r}
\Delta u(x)=0 \text { for } x \in \Omega_{3}^{c} \\
\lim _{\substack{x \rightarrow p \\
x \in \Omega_{3}^{c}}} \frac{\partial u}{\partial \nu}(x)=f(p) \text { for } p \in \partial \Omega_{3},
\end{array}
$$

where $\Omega_{3}$ is the domain shown in Figure $5 \mathrm{c}, \frac{\partial}{\partial \nu}$ denotes differentiation with respect to the outward pointing normal vector, and the boundary data $f(p)$ was taken to be the outward normal derivative on $\partial \Omega_{3}$ of a potential function $u$ arising from 5 charges placed randomly in the interior of $\Omega_{3}$. The solution $u(x)$ of $(7.1)$ was represented in the form

$$
u(x)=\frac{1}{2 \pi} \int_{\partial \Omega_{3}} \log |x-y| \sigma(y) d S(y) .
$$

A total of 871 quadratures nodes were used to discretize the resulting boundary integral equation

$$
\frac{1}{2} \sigma(x)+\frac{1}{2 \pi} \int_{\partial \Omega_{3}} \nabla_{x} \log |x-y| \cdot \nu(x) \sigma(y) d S(y)=f(x) .
$$

The 12 corner regions were discretized using purpose-made quadratures, which ranged in size from 17 to 21 nodes, while the smooth portions of the curve were discretized using 630 piecewise Legendre quadrature nodes. Figure 3 shows four charge basis functions obtained in the process of constructing a purpose-made quadrature for one of the corner regions.

In order to access the accuracy of the discretization, the difference between the true potential function $v$ and the single layer potential

$$
u(x)=\frac{1}{2 \pi} \int_{\partial \Omega_{3}} \log |x-y| \sigma(y) d S(y),
$$

where $\sigma$ is the computed inverse of equation (7.3), was measured at a collection of 100 randomly chosen points in the exterior of the domain $\Omega_{3}$ as well as at 300 points on a circle $\Gamma$ enclosing the domain $\Omega_{3}$. The largest absolute error was found to be $1.78 \times 10^{-13}$ and the estimated relative $L^{2}(\Gamma)$ error $\|u-v\|_{2} /\|v\|_{2}$ was $6.24 \times 10^{-13}$. 


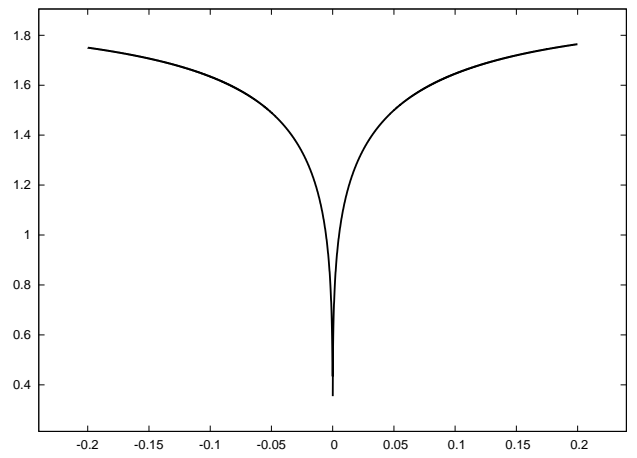

(a)

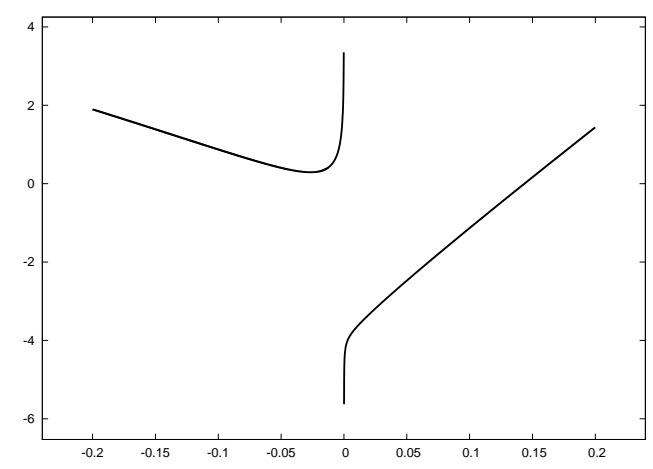

(c)

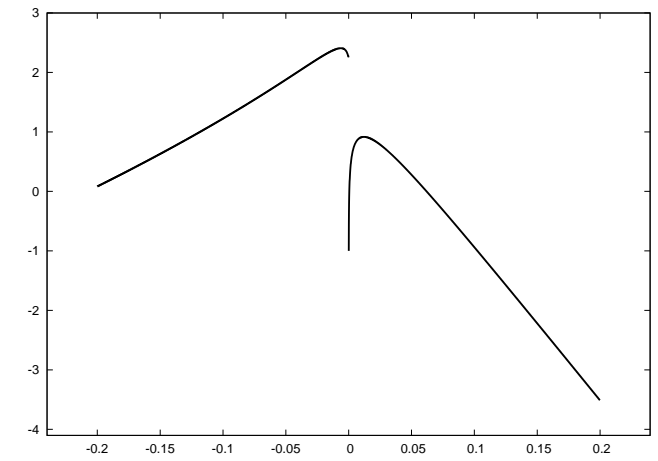

(b)

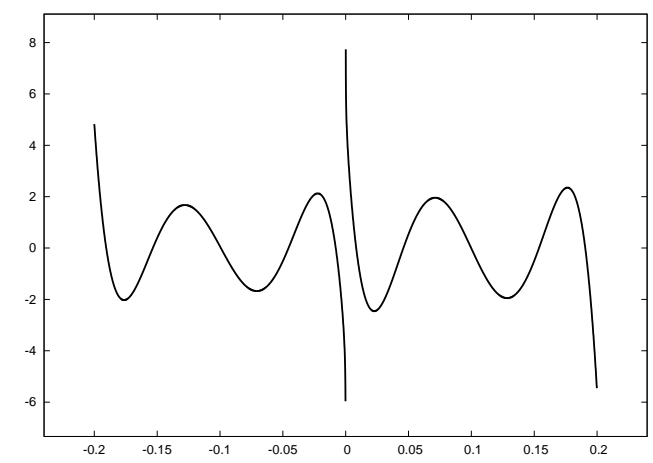

(d)

Figure 3: Four charge basis functions used in the discretization of a Laplace Neumann boundary integral equation on the contour $\partial \Omega_{3}$.

7.1.2. A Helmholtz Dirichlet problem. In this example, we solved the Helmholtz exterior Dirichlet problem

$$
\begin{gathered}
\Delta u(x)+k^{2} u(x)=0 \text { for } x \in \Omega^{c} \\
\lim _{\substack{x \rightarrow p \\
x \in \Omega^{c}}} u(x)=f(p) \text { for } x \in \Omega^{c},
\end{gathered}
$$

where $\Omega$ is the "shark's fin" domain shown in Figure 4, the wavenumber $k$ is 20 , and $f(p)$ is taken to be the restriction to the boundary $\partial \Omega$ of a acoustic potential $u(x)$ generated by 5 random charges placed in the interior of the domain $\Omega$. The domain $\Omega$ is approximately 80 wavelengths by 80 wavelengths in size.

The solution $u(x)$ of the problem (7.4) was represented in the form

$$
u(x)=\int_{\partial \Omega}\left(\frac{i}{4} \nu(y) \cdot \nabla_{y}+1\right) H_{0}(k|x-y|) \sigma(y) d S(y),
$$

where $\nu(y)$ denotes the outward pointing normal vector to the contour $\partial \Omega$ at the point $y, \nabla_{y}$ denotes the gradient in the $y$ variable, and $H_{0}$ is the Hankel function of the first kind of order 0 . This representation leads to the boundary integral equation

$$
-\frac{1}{2} \sigma(x)+\int_{\partial \Omega}\left(\frac{i}{4} \nu(y) \cdot \nabla_{y}+1\right) H_{0}(k|x-y|) \sigma(y) d S(y)=f(x) .
$$

This integral equation was discretized using a combination purpose-made quadratures, one for each of the 3 corner regions of $\partial \Omega$, and 30-point piecewise Legendre quadratures for the smooth portions of the contour. A total of 1163 quadratures nodes were used; the purpose-made quadratures were 70, 68, and 
65 points. The error in the representation (7.5) was measured at 100 random points in the exterior of

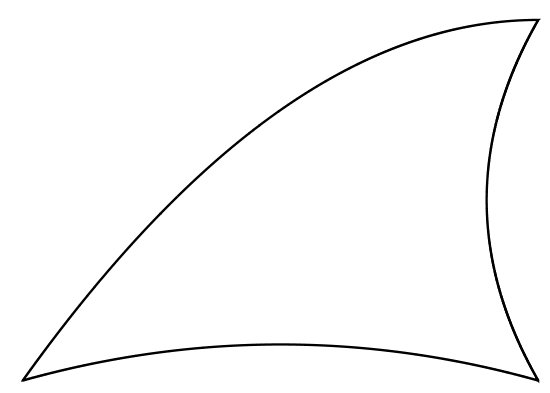

Figure 4: The "shark's fin" domain $\Omega$ under consideration in Section 7.1.2.

the domain $\Omega$ as well as at 300 points on a circle $\Gamma$ enclosing $\Omega$. The largest absolute error was found to be $3.56 \times 10^{-13}$ while the estimated relative $L^{2}(\Gamma)$ error was found to be $7.67 \times 10^{-12}$.

7.2. Universal quadratures. Using the procedures described in Sections 5 and 6, universal quadratures were constructed for the discretization over domains with corners of the boundary integral equations

$$
\begin{gathered}
-\frac{1}{2} \sigma(x)+\frac{1}{2 \pi} \int_{\partial \Omega}\left(\nabla_{y} \log |x-y| \cdot \nu(y)+1\right) \sigma(y) d S(y)=u(x), \\
\frac{1}{2} \sigma(x)+\frac{1}{2 \pi} \int_{\partial \Omega} \nabla_{x} \log |x-y| \cdot \nu(y) \sigma(y) d S(y)=u(x),
\end{gathered}
$$

and

$$
-\frac{1}{2} \sigma(x)+\int_{\partial \Omega}\left(\frac{i}{4} \nu(y) \cdot \nabla_{y}+1\right) H_{0}(k|x-y|) \sigma(y) d S(y)=u(x),
$$

where $H_{0}$ is the Hankel function of zeroth order, $\nu(y)$ denotes the outward pointing unit normal vector of $\partial \Omega$ at the point $y$. Several collections of quadratures were constructed for the boundary integral equation (7.9), one for each of several different ranges of the wavenumbers $k$ in the interval [.1, 20]. The integral equation (7.7) arises from the solution of the exterior Dirichlet problem for Laplace's equation, (7.8) arises from the solution of the exterior Neumann problem for Laplace's equation, and (7.9) is associated with the exterior Dirichlet problem for the Helmholtz equation with wavenumber $k$ (see [5], for instance).

\begin{tabular}{llllcccc}
\hline & \multicolumn{9}{c}{$\Omega_{1}$} & \multicolumn{3}{c}{$\Omega_{2}$} \\
\hline Problem & $\mathrm{k}$ & $N$ & $T$ & $E_{\text {pot }}$ & $\mathrm{N}$ & $T$ & $E_{\text {pot }}$ \\
\hline Laplace Dirichlet & - & 258 & 0.02 & $9.90 \times 10^{-12}$ & 1320 & 0.31 & $4.86 \times 10^{-13}$ \\
Laplace Neumann & - & 351 & 0.02 & $3.80 \times 10^{-14}$ & 1376 & 1.45 & $4.72 \times 10^{-12}$ \\
Helmholtz Dirichlet & 0.1 & 364 & 0.71 & $1.11 \times 10^{-13}$ & 1512 & 4.82 & $1.72 \times 10^{-12}$ \\
& 0.5 & 370 & 0.74 & $6.90 \times 10^{-14}$ & 1528 & 5.12 & $3.55 \times 10^{-12}$ \\
& 1 & 375 & 0.79 & $7.75 \times 10^{-14}$ & 1532 & 5.19 & $7.87 \times 10^{-12}$ \\
& 2 & 380 & 0.83 & $1.38 \times 10^{-13}$ & 2504 & 10.76 & $6.24 \times 10^{-14}$ \\
& 5 & 570 & 1.35 & $5.57 \times 10^{-12}$ & 2532 & 11.52 & $2.33 \times 10^{-13}$ \\
& 10 & 572 & 1.49 & $7.98 \times 10^{-14}$ & 2552 & 14.79 & $1.75 \times 10^{-11}$ \\
& 20 & 939 & 2.59 & $7.81 \times 10^{-14}$ & 4492 & 31.23 & $6.75 \times 10^{-12}$ \\
\hline
\end{tabular}

Table 1: Computational results obtained for the Pacman and inkblot domains $\Omega_{1}$ and $\Omega_{2}$. 


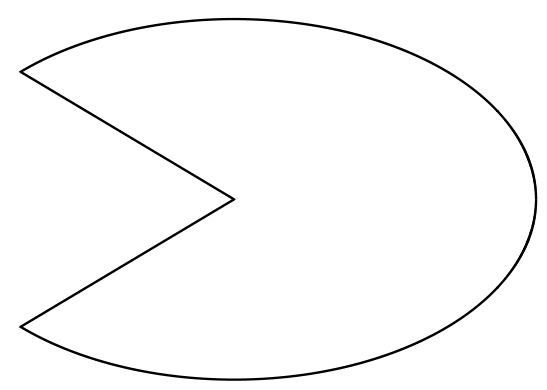

(a) The "Pacman" domain $\Omega_{1}$.

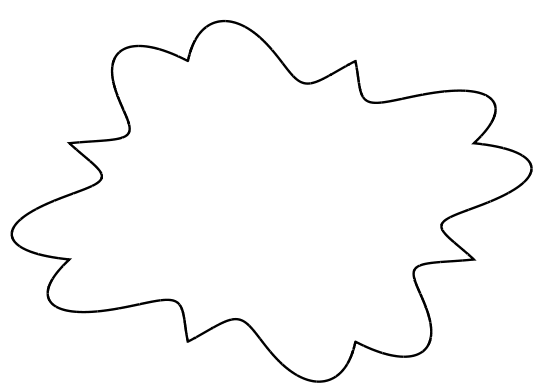

(b) The "inkblot" domain $\Omega_{2}$.

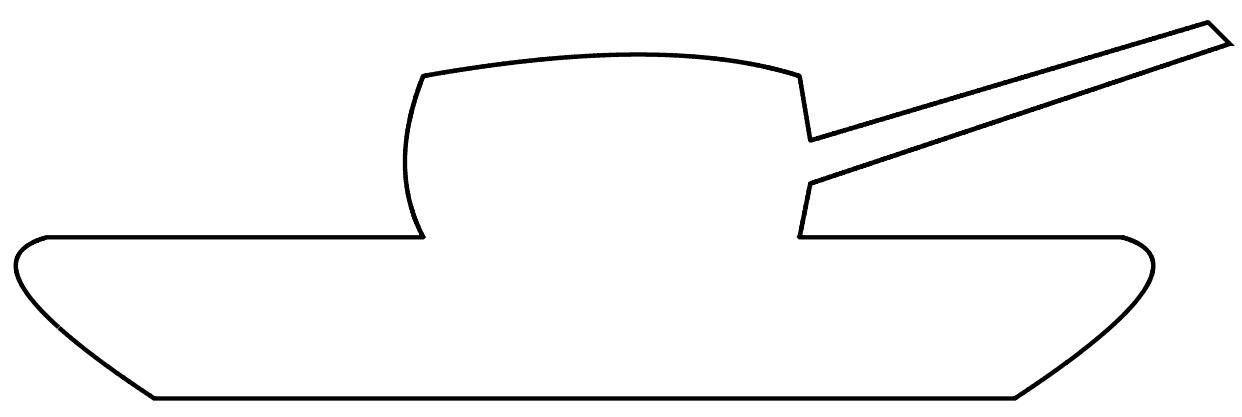

(c) The "tank" domain $\Omega_{3}$.

Figure 5: The domains under consideration in Section 7.2. The Pacman domain is approximately 4 units by 4 units in size, while the dimensions of the inkblot domain are approximately 6 by 6 . The tank domain is approximately 6 units in length by 3 units in height.

\begin{tabular}{llccc}
\hline & & \multicolumn{3}{c}{$\Omega_{3}$} \\
\hline Problem & $\mathrm{k}$ & $N$ & $T$ & $E_{\text {pot }}$ \\
\hline Laplace Dirichlet & - & 1197 & 0.76 & $9.10 \times 10^{-12}$ \\
Laplace Neumann & - & 1385 & 1.50 & $4.62 \times 10^{-12}$ \\
Helmholtz Dirichlet & 0.1 & 1359 & 8.73 & $2.54 \times 10^{-13}$ \\
& 0.5 & 1385 & 9.29 & $4.82 \times 10^{-13}$ \\
& 1 & 1403 & 9.61 & $1.11 \times 10^{-12}$ \\
& 2 & 1992 & 14.74 & $1.30 \times 10^{-13}$ \\
& 5 & 2746 & 25.49 & $1.13 \times 10^{-13}$ \\
& 10 & 2777 & 26.71 & $7.59 \times 10^{-13}$ \\
& 20 & 3221 & 30.94 & $3.70 \times 10^{-13}$ \\
\hline
\end{tabular}

Table 2: Computational results obtained for the domain $\Omega_{3}$

For each of the domains shown in Figure 5, the exterior Dirichlet and Neumann problems for Laplace's equation and the exterior Dirichlet problem for the Helmholtz equation at various wavenumbers were solved. In each case, the boundary data was taken to be an electromagnetic or acoustic potential generated by a collection of 5 random charges in the interior of the domain and the boundary integral equation was discretized using universal quadratures for corner regions and piecewise Legendre quadratures on the smooth portions of the curve. The resulting solution was tested by comparing the value of 
the true potential function to the computed solution at 100 randomly chosen points in the exterior of the domain. Tables 1 and 2 present the results. The following quantities are reported for each problem:

- $\mathrm{k}$ the wavenumber for the problem (if applicable);

- $N$ is the number of discretization nodes required for the problem;

- $T$ is the wall clock time required to solve the integral equation; and

- $E_{\text {pot }}$ the largest absolute error measured in the computed potential.

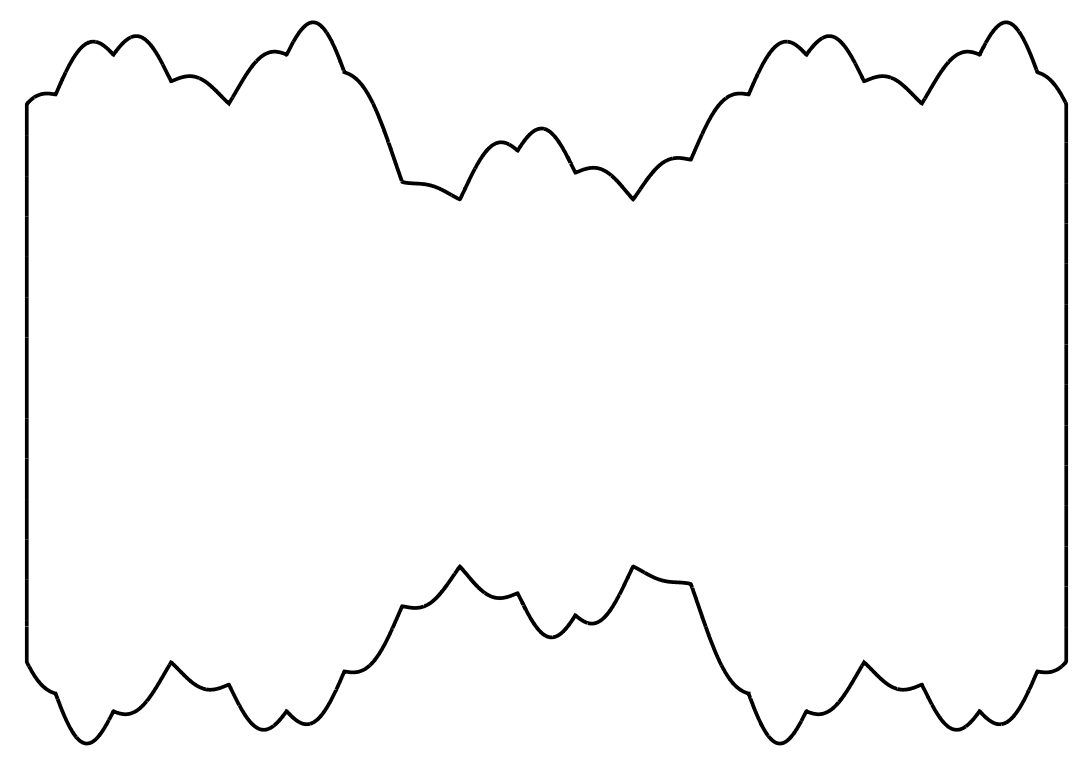

Figure 6: The domain $\Omega_{4}$, formed from the graph of two Lipschitz functions.

7.3. A final example. As a final example, we used universal quadratures to solve an exterior Dirichlet problem for the Helmholtz equation at wavenumber $k=1$ on the domain $\Omega_{4}$ shown in Figure 6 . The boundary of $\Omega_{4}$ is formed from two Lipschitz functions and has 40 corner points. The domain $\Omega_{4}$ is approximately 3 wavelengths in width and 3 wavelengths in height. The boundary data for the problem was taken to be an acoustic potential generated by 5 charges randomly placed in the interior of the domain.

The discretization of the boundary integral equation (7.9), which was used to solve the boundary value problem, required 7794 discretization nodes. Between 54 and 70 discretization nodes were required per corner. Inversion of the resulting discrete linear system required 71.83 seconds and resulting in an approximate charge distributions which, when compared with the true potential function at a collection of 100 randomly placed points in the exterior of the domain, yielded a maximum absolute error of $2.13 \times 10^{-12}$.

\section{Conclusions}

We have introduced an algorithm for the construction of "universal quadratures" for the efficient discretization of Laplace and Helmholtz boundary integral equations over a very general class of domains with corner points. Once they have been constructed, these quadratures reduce the complexity of solving a boundary integral equation on such domains to approximately that of solving the same equation over well-behaved smooth domains. While the implementation presented here is for certain Laplace and 
Helmholtz boundary value problems over planar domains with corners, the extension of our approach to other boundary value problems, other pathological domains, and to surfaces with singularities is straightforward and will be reported at a later date.

\section{ACKNOWLEDGMENTS}

The first and third authors were supported by the Office of Naval Research under contract N0001409-1-0318. The second author was supported in part by the ONR under contract N0014-07-1-0711, in part by the AFOSR under contract FA9550-09 -1-0241, and in part by DARPA-AFOSR contract FA9550-07-1-0541.

\section{REFERENCES}

[1] K. Atkinson, The Numerical Solution of Integral Equations of the Second Kind, Cambridge University Press, 1997.

[2] A. BJörck, Numerical Methods for Least Squares Problems, SIAM, Philadephia, 1996.

[3] J. Bremer, Z. Gimbutas, and V. Rokhlin, A nonlinear optimization procedure for generalized Gaussian quadratures, Yale University, Department of Computer Science Tech Report TR1406, 2008.

[4] J. Bremer And V. Rokhlin, Efficient discretization of Laplace boundary integral equations on polygonal domains, Journal of Computational Physics (to appear).

[5] D. Colton And R. Kress, Inverse Acoustic and Electromagnetic Scattering Theory, Springer-Verlag, 1992.

[6] L. Greengard, P. Martinsson, D. Gueyffier, and V. Rokhlin, Fast direct solvers for integral equations in complex three-dimensional domains, Acta Numerica, (2008), pp. 1-33.

[7] P. Grisvard, Singularities in Boundary Value Problems, Springer-Verlag, 1992.

[8] M. Gu AND S. EISEnStAT, Efficient algorithms for computing a strong rank-revealing QR factorization, SIAM J. Sci. Comput., 17 (1996), pp. 848-869.

[9] J. Helsing And R. OJala, Corner singularities for elliptic problems: integral equations, graded meshes, quadrature, and compressed inverse preconditioning, Journal of Computational Physics, 227 (2008), pp. 8820-8840.

[10] N. Koshliakov, M. Smirnov, And E. B. Gliner, Differential Equations of Mathematical Physics, North-Holland, Amsterdam, 1964. 